Front end and opt i cs of i nfrared beami ne at SPr i ng- 8

\begin{tabular}{|l|l|}
\hline 著者 & $\begin{array}{l}\text { K MURA S, K MURA H, TAKAHASH T, FUKUI K, } \\
\text { KONDO Y, YOSH MATSU Y, MORI WAK T, NANBA T, } \\
\text { I SH KAMA T }\end{array}$ \\
\hline $\begin{array}{l}\text { j our nal or } \\
\text { publ i cat i on t it l e }\end{array}$ & $\begin{array}{l}\text { Nucl ear I nst r ument s and Met hods i n Physi cs } \\
\text { Resear ch Sect i on A }\end{array}$ \\
\hline vol une & $467-468$ \\
\hline number & 1 \\
\hline page r ange & $437-440$ \\
\hline year & $2001-07$ \\
\hline URL & ht t p: //hdl . handl e. net /10098/1566 \\
\hline
\end{tabular}




\title{
Front end and optics of infrared beamline at SPring-8
}

\author{
S. Kimura ${ }^{\text {a,b, , , H. Kimura }}{ }^{\mathrm{c}}$, T. Takahashid ${ }^{\mathrm{d}}$, K. Fukui ${ }^{\mathrm{e}}$, Y. Kondo ${ }^{\mathrm{f}}$, \\ Y. Yoshimatsu ${ }^{\mathrm{f}}$, T. Moriwaki, T. Nanba ${ }^{\mathrm{a}}$, T. Ishikawa ${ }^{\mathrm{g}}$ \\ "Graduate School of Science and Technology, Kobe University. Rokkodai-cho. Nadu-ku, Kobe 657-8501, Japan \\ ${ }^{b}$ PRESTO, Japan Science and Technologl Corporation, Japan \\ 'SPring-8/JASRI. Mikazuki, Hvogo 679-5198. Japan \\ ${ }^{d}$ Research Reacror Institule. Kuoto Universit. Osaka 590-0494. Japan \\ enstitute for Molecular Science, Okazaki 444-8585, Japan \\ 'Department of Applied Physics, Tohoku Unilersits. Sendai 980-8579. Japan \\ "SPring-8/RIKEN, Mikazuki, Hyogo 679-5/48. Japan
}

\section{Abstract}

An infrared (IR) beamline, BL43IR, at SPring-8 has been constructed for IR spectroscopies under extreme conditions. The beamline covers a very wide photon energy range in the IR region and offers a brilliant IR photon beam to the experimental end stations. The IR light from SPring- 8 is collected by a newly designed optical system including a so-called "magic mirror". The design of the optical system and the obtained results are reported.

Keywords: Infrared synchrotron radiation; Optical system; Magic mirror; SPring- 8

\section{Introduction}

SPring-8 is a third generation X-ray light source and the largest storage ring for synchrotron radiation in the world. The construction plan of an infrared (IR) beamline was proposed about eight years ago, because the vertical divergence of the $I R$ beam is much lower than for a small SR light source, for instance, UVSOR [1]. The IR beamline, BL43IR, was dedicated for materials research from FY2000. The optical system including a so-called "magic mirror" was designed and installed as a new concept of a collection mirror of the light emitted from the long emission length of the bending magnet. In this paper, the design of the optical system and the first commissioning results (beam size and brilliance) are reported. 


\section{Optical system}

The beamline was designed to use IR synchrotron radiation (IRSR) from a bending magnet with the acceptance angle of 36.5 (horizontal) $\times 12.6$ (vertical) $\mathrm{mrad}^{2}$. Since the radius of the electron beam orbit is $39.3 \mathrm{~m}$ at the bending magnet, the emission length is $1.44 \mathrm{~m}$. To focus the light from this long source, we employed a perfect focusing mirror for the circular orbit, a so-called "magic mirror" proposed by LopezDelgado and Szwarc [2]. The magic mirror not only focuses the source but also reduces the time delay due to the large acceptance: the summation of the optical path length and the electron orbital length is the same for each emitting position. We have expanded the formula to include the vertical direction. The vertical direction is approximated by a spherical curve using the distance between the emission and focusing points and the incident angle to the magic mirror. The formula of the coordinates of the surface of the magic mirror $(M)$ is

$$
\begin{aligned}
& \overline{A M}(\theta)=\frac{\frac{1}{2}\left\{\left(d_{0}-\rho \theta\right)^{2}-\rho^{2}-a^{2}\right\}+a \rho \sin \theta}{d_{0}-\rho \theta-a \cos \theta}, \\
& \overline{M I}(\theta)=\sqrt{\rho^{2}+\overline{A M}(\theta)^{2}+a^{2}-2 a(\rho \sin \theta+\overline{A M}(\theta) \cos \theta),} \\
& \left.R(\theta)=\frac{2 \overline{A M}(\theta)\left(d_{0}-\rho \theta-\overline{A M}(\theta)\right)}{d_{0}-\rho \theta} \cos \left[\frac{1}{2} \cos ^{-1 / \overline{A M}(\theta)-a \cos \theta}\right)\right\rceil,
\end{aligned}
$$

and

$$
\left\{\begin{aligned}
x(\theta, v)= & \rho \cos \theta-\overline{A M}(\theta) \sin \theta+\frac{|R(\theta)|\{\sin \theta-(\rho \cos \theta-\overline{A M}(\theta) \sin \theta) / \bar{M}(\theta)\}}{\sqrt{2+2(\overline{A M}(\theta)-a \cos \theta) / \overline{M I}(\theta)}} \\
& \times\left(1-\cos \left(\tan ^{-1} \frac{v}{R(\theta)}\right)\right\}, \\
y(\theta, v)= & \rho \sin \theta+\overline{A M}(\theta) \cos \theta+\frac{|R(\theta)|\{-\cos \theta+(a-\rho \sin \theta-\overline{A M}(\theta) \cos \theta) / \bar{M}(\theta)\}}{\sqrt{2+2(\overline{A M}(\theta)-a \cos \theta) / \overline{M I}(\theta)}} \\
& \times\left(1-\cos ^{\prime}\left(\tan ^{-1} \frac{v}{R(\theta)}\right) \mid,\right. \\
z(0, v)= & R(\theta) \sin \left(\tan ^{-1} \frac{v}{R(\theta)}\right) .
\end{aligned}\right.
$$

Here, $\rho$ is the radius of the electron orbit, a the length between the center of the electron orbit and the focal point, $d_{0}$ the optical pass from a standard point, $\theta$ the acceptance angle of SR from electron orbit and $v$ the vertical size of the magic mirror. The parameters for $B L 43 \mathbb{R}$ are $\rho=39271.8 \mathrm{~mm}, a=46025.4 \mathrm{~mm}$, $d_{0}=69807.4 \mathrm{~mm}, \theta=1.4804-1.5169 \mathrm{rad}$ and $v=-22-22 \mathrm{~mm} . \overline{A M}, \overline{M I}$ and $R$ have the same meanings as in ref. [2].

The schematic figure of the BL43IR optical system is shown in Fig. 1. The magic mirror (M1) is located at $4.37 \mathrm{~m}$ from the center of the emission point. The focal point is located at $11.32 \mathrm{~m}$ from the center of the emission point. The result of the ray trace at the focusing point is shown in Fig. 2(a). The beam size at the focal point depends on the electron beam size $\left(\sigma: 200(\mathrm{~h}) \times 20(\mathrm{v}) \mu \mathrm{m}^{2}\right.$ was assumed) and the emission angle of the IRSR. The emission angle also depends on photon energy. Fig. 2(a) is the result at $h v=0.5 \mathrm{eV}$ $\left(\sigma^{\prime} \sim 2.5 \mathrm{mrad}\right)$. The energy is at the center of the range covered by a HgCdTe detector, which is used in the beam size measurement. The beam size $(\sigma)$ at the focal point is $0.63(\mathrm{~h}) \times 0.37(\mathrm{v}) \mathrm{mm}^{2}$. In the calculation, the diffraction effect was not included because the diffaction effect is negligible at $h v \geqslant 0.04 \mathrm{eV}$. 


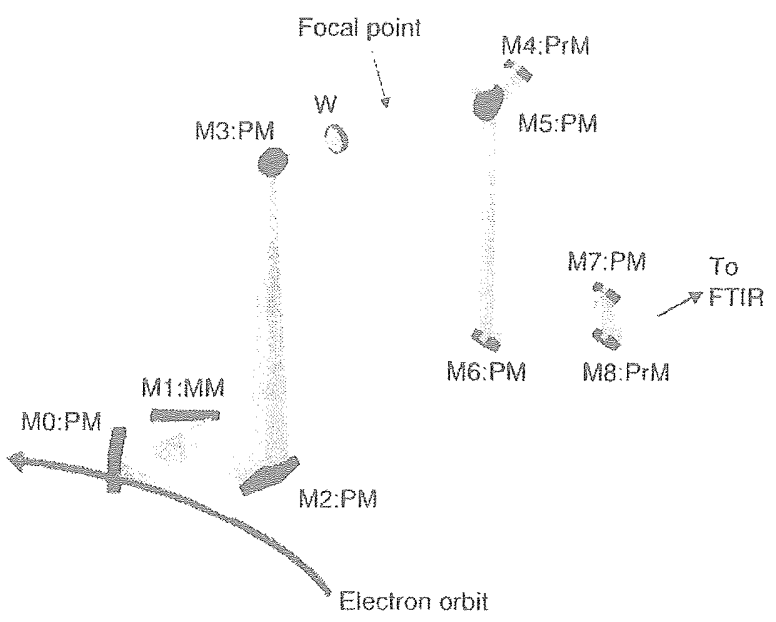

Fig. 1. Schematic figure of the optical system of the infrared beamline at SPring-8. PM. MM and PrM indicate plane, magic and offaxis parabolic mirrors. respectively. W is an optical window, which separates upstream ultrahigh-iacuum area and downstream lowvacuum one. The two optical windows (wedged-diamond and $\mathrm{BaF}_{2}$ ) are installed and can be changed without breaking vacuum.
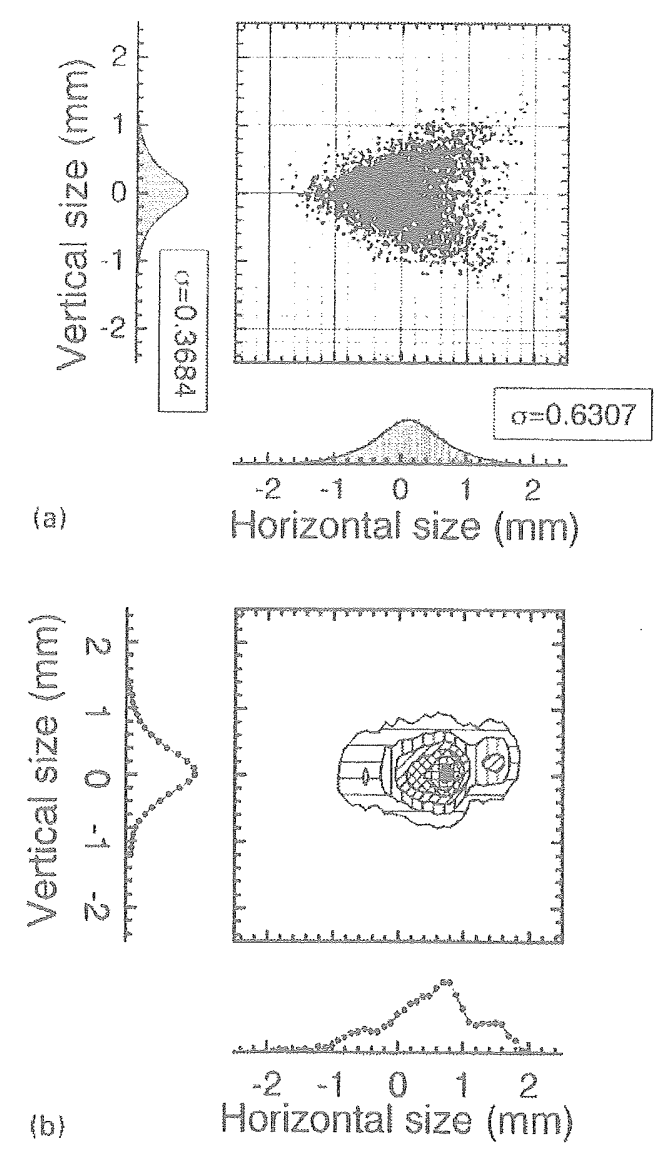

Fig. 2. Calculated photon beam image of $h v=0.5 \mathrm{eV}\left(\sigma^{1}-2.5 \mathrm{mrad}\right)$ (a) and measured beam profile by a HgCdTe detector (b) at the focal point in Fig. 1 . 


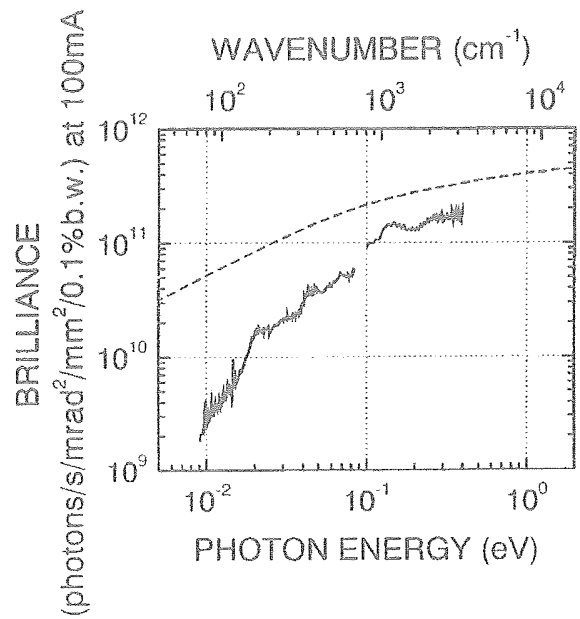

Fig. 3. Calculated (dashed hne) and measured (solid line) brilliance of BL43IR

\section{Observed bean size and brilliance}

In order to investigate the performance of the magic mirror, the cross-sectional size of SR was measured at the focal point. The IRSR of $h=0.05-1 \mathrm{eV}$ was detected using a HgCdTe detector with an entrance aperture of $100 \mu \mathrm{m}$ diameter by moving the detector stage. Fig. $2(\mathrm{~b})$ shows the observed spot size, where "horizontal" means the component parallel to the orbital plane of the electron beam. The root-meansquare spreads of the Gaussian line shape $\left(\sigma_{x, y}\right)$ fitted to the horizontal and verical components are 0.63 and $0.37 \mathrm{~mm}$, respectively. The beam size is in good agreement with the results of the ray trace.

Fig. 3 shows the measured (solid line) and calculated (dashed line) brilliance of IRSR. Here, the optical emitance was calculated from the acceptance angle and the observed beam size of SR at the focal point as shown in Fig. 2. The observed brilliance is smaller than the calculated one. The reason is due to the loss by optical components and/or the evaluation error of the beam size and the divergence at the focal point.

In the far-infrared region the observed intensity more rapidly decreases on the long-wavelength side in comparison with the calculated ones. This originates from the difraction effect caused by the firite size of the optical components.

\section{Summary}

The performance of the $\mathrm{R}$ beamline $\mathrm{PL} 43 \mathrm{R}$ of SPring-8 was reported. A magic mirror" can efficiently focus the source in spite of the long emission length and consequently works very well in suppling a very narrow photon beam to each experimental end station of the beam line.

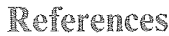

I1] M. Hara, S.H. Be, I. Takeshita, T. Nanba, Rev. Sci. Instrum. 63 (1992) 1543.

[2] R. Lopez.Delgado, H. Szwarc, Opt. Commun. 19 (1976) 286. 